\title{
Whole genome bisulfite sequencing reveals a sparse, but robust pattern of DNA methylation in the Dictyostelium discoideum genome.
}

\author{
Jacob L. Steenwyk ${ }^{1,3}$, James St. Denis ${ }^{1}$, Jacqueline M. Dresch ${ }^{2}$, \\ Denis A. Larochelle ${ }^{1}$ and Robert A. Drewell ${ }^{1}$ \\ ${ }^{1}$ Biology Department, Clark University, 950 Main Street, Worcester, MA 01610 \\ 2 Department of Mathematics and Computer Science, Clark University, 950 Main Street, \\ Worcester, MA 01610 \\ ${ }^{3}$ Department of Biological Sciences, Vanderbilt University, Box 351634 Station B, Nashville, TN \\ 37235
}

Corresponding author: Robert A. Drewell

Tel: +15087937625

Fax: +1 5087937174

Short title: DNA methylation in Dictyostelium

Keywords: DNA methylation, Dictyostelium, methylome, epigenetics 


\begin{abstract}
DNA methylation, the addition of a methyl $\left(\mathrm{CH}_{3}\right)$ group to a cytosine residue, is an evolutionarily conserved epigenetic mark involved in a number of different biological functions in eukaryotes, including transcriptional regulation, chromatin structural organization, cellular differentiation and development. In the slime mold Dictyostelium, previous studies have shown the existence of a DNA methyltransferase (DNMA) belonging to the DNMT2 family, but the extent and function of 5-methyl-cytosine in the genome is unclear. Here we present the whole genome DNA methylation profile of Dictyostelium discoideum using deep coverage, replicate sequencing of bisulfite converted gDNA extracted from post-starvation cells. We find an overall very low level of DNA methylation, occurring at only 462 out of the 7.5 million $(0.006 \%)$ cytosines in the genome. Despite this sparse profile, significant methylation can be detected at 51 of these sites in replicate experiments, suggesting they are robust targets for DNA methylation. These 5methyl-cytosines are associated with a broad range of protein-coding genes, tRNA-encoding genes and retrotransposable elements. Our data provides evidence of a minimal, but functional, methylome in Dictyostelium, thereby making Dictyostelium a candidate model organism to further investigate the evolutionary function of DNA methylation.
\end{abstract}




\section{Introduction}

DNA methylation is a post-synthetic modification that typically occurs on cytosine residues in eukaryotic plants and animals [1]. Generally, DNA methylation is associated with transcriptional repression [2], but has been linked to more complex processes including cell differentiation [3], genomic imprinting and stability $[4,5]$, and $X$ chromosome inactivation [6]. The majority of DNA methylation is carried out by the DNA methyltransferases DNMT1, DNMT3A and DNMT3B [7]. DNMT1 is responsible for maintaining a cell's methylation profile post-replication by targeting hemi-methylated sites in the genome [8], while DNMT3A and DNMT3B methylate CPG dinucleotides de novo, thereby creating new epigenetic marks [9]. Although DNA methylation appears to be evolutionarily conserved across a large number of eukaryotes (Fig. 1), some organisms, such as Dictyostelium discoideum and Drosophila melanogaster, lack the major DNMTs while retaining the less characterized methyltransferase DNMT2 [8].

DNMT2 was originally identified based on sequence conservation of essential catalytic motifs and exhibits structural similarity to other DNMTs $[10,11]$. It is found as a single copy gene across the eukaryotic tree of life in protists, plants, fungi and animals (Fig. 1), suggesting an important functional role [11]. To date, DNMT2 has been shown to methylate tRNAs [12] and contribute to DNA methylation of retrotransposons in D. melanogaster [13], demonstrating a dual specificity for DNA and RNA substrates [14]. Despite these observations, characterizing the presence, relevance and efficacy of DNA methylation in organisms containing only a DNMT2 methyltransferase remains an active area of research [10, 11, 14-16].

Dictyostelium discoideum, a eukaryotic slime mold containing a Dnmt2-homolog (dnmA) $D D B 0231095)[17,18]$, is among the organisms where the presence, extent and function of DNA methylation is debated. In the initial 1991 study, D. discoideum was reported to lack DNA methylation, as determined by whole genome methylation-sensitive restriction enzyme analysis and high performance liquid chromatography assays [19]. Despite this early report, the confirmed existence of a DNMT2 homolog, along with D. discoideum's unique AT-rich genome and an under-representation of $\mathrm{CpG}$ dinucleotides relative to the $\mathrm{GpC}$ isomer, implied the possible presence of a DNA methylation system, as methylated $\mathrm{CpGs}$ are inherently chemically unstable and readily mutate to TpGs [17]. Accordingly, the investigation of DNA methylation in 
D. discoideum was revisited in 2006 using more advanced methodologies including antibodies to detect 5-methylcytosine in bulk genomic DNA across distinct developmental time points [20]. These studies detected overall low levels of DNA methylation in the genome and were confirmed using methylation-sensitive restriction digests targeted to retrotransposons and several other genes [20]. A functional role for the DNMA enzyme in silencing of retrotransposons via the asymmetric methylation of cytosine residues was confirmed using bisulfite sequencing of specific sites [18]. Furthermore, DNA methylation was demonstrated to increase through development (with the highest levels at 24 hours post-starvation) and knocking out $d n m A$ revealed developmental defects and reduced DNA methylation [20]. More recently, detailed studies examining the activity of the $D$. discoideum DNMA enzyme demonstrated that specific tRNA molecules are the preferred target substrate for this methyltransferase, but other substrates potentially remain to be characterized [21]. These conflicting reports, coupled to the relatively few studies investigating DNA methylation in $D$. discoideum, therefore reflect the uncertainty regarding the status of the DNA methylation system in this species.

To determine if a functioning DNA methylation system is present in D. discoideum, we utilized whole genome bisulfite sequencing [22] to give deep coverage ( $435 x)$ of genomic DNA isolated from cells in an 18-24 hours developmental time window. Our results show that $D$. discoideum harbors a minimal, but replicable methylome. Overall, cytosine methylation is sparse in the $D$. discoideum genome, occurring at only $0.006 \%$ of all sites (462 out of the 7.5 million cytosines in the genome), many of which demonstrate low levels of methylation. Despite this profile, significant methylation can be consistently detected at 51 of these sites in replicate experiments, suggesting they are robust targets for DNA methylation. These 5-methylcytosines are associated with a broad range of protein-coding genes, tRNA-encoding genes and retrotransposable elements. Taken together, our studies provide evidence of a minimal, but functional, methylome in $D$. discoideum, thereby making $D$. discoideum a candidate model organism to further investigate the enzymatic role of Dnmt2 and the evolutionary function of DNA methylation. 


\section{Results and Discussion}

\section{Mapping, conversion and coverage rates}

Sequencing of bisulfite-converted genomic DNA from Dictyostelium discoideum AX4 cells grown to between 18 and 24 hours of development, and spike-in control lambda bacteriophage DNA, generated 125 million reads after quality control (see Methods for details). This time window for the cells was chosen to ensure temporal heterogeneity of the gDNA sample and reflects the fact that previous studies have reported higher levels of DNA methylation in cells 24 hours poststarvation [20]. Of the sequencing reads, $72.53 \%$ mapped to unique regions of the $D$. discoideum genome, which equates to $286 x$ coverage across the entire genome. More than $90 \%$ of the bases in the genome are covered by 100 or more reads (Fig. $2 \mathrm{a}$ and b). Over $80 \%$ of cytosines are covered by 75 or more reads (Fig. 2c), resulting in coverage of $98.816 \%$ of all cytosines in the genome (Table S1) with very similar profiles for CG, CHG and $\mathrm{CHH}$ sequence context (Fig. 2c). The bisulfite conversion rate in our experiment was $99.44 \%$, measured by the $\mathrm{C}$-to- $\mathrm{U}$ deamination rate in the unmethylated lambda genome, indicating that the false-negative rate was less than $0.6 \%$.

\section{Overall genomic methylation profile}

A very small proportion of cytosines in the Dictyostelium genome are methylated. The average overall level of methylation across the entire genome, calculated from the ratio of $\mathrm{C}$ reads to total reads (see Methods for details), is only $0.523 \%$, with a similar profile across all chromosomes and mitochondrial DNA (Table 1). It should be noted that this may include a number of false positive $\mathrm{C}$ reads arising from a failure to convert in the bisulfite reaction and therefore is likely an overestimate of the global methylation level. Nonetheless, methylation is detected predominantly at $\mathrm{CHH}$ sequences (where $\mathrm{H}=$ non- $\mathrm{G}$ base), but is also found at $\mathrm{CG}$ and CHG sequences (Table 2). Of the approximately 7.5 million cytosines in the genome, only 462 ( $0.006 \%$ of all sites) are significantly methylated, of which only 27 are CpG sites (Table 2 ). This methylome profile mirrors previous studies that have reported global methylation levels in the Dictyostelium genome of $0 \%$ [19], $0.14 \%$ [20], and $0.20 \%$ [18] using different methodologies, indicating that DNA methylation is very rare in this species. These results are strikingly different from the methylation profile observed in other eukaryotes. In mammals, $60-90 \%$ of $\mathrm{CpGs}$ can be methylated [23], while in hymenoptera insects $0.51-0.67 \%$ of $\mathrm{CpGs}$ are methylated [24, 25]. The 
sparse $0.006 \%$ of methyl-CpGs detected in Dictyostelium is therefore at least two orders of magnitude lower than the level found in other eukaryotic organisms. Among the individual sites that demonstrate a significant level of methylation in our study, the vast majority are methylated in less than $10 \%$ of reads, but cytosines with up to a $100 \%$ methylation level are detected (Fig. 3).

\section{Methylation levels at individual mCs}

In order to further investigate the methylation level at the 462 individual cytosine residues we analyzed their distribution across the genome. All chromosomes and the mitochondrial DNA show a wide range of methylation levels for $\mathrm{mCs}$, with $\mathrm{mCHH}$ sites carrying the broadest range (Fig. 4a). In parallel, we plotted the total read number for each of these mCs (Fig. 4b) against methylation level. Strikingly, there is a clear inverse relationship between read depth and methylation level at the $\mathrm{mCs}$, with sites showing higher levels of methylation having lower read depth and vice-versa (Fig. 4c and d). This indicates that the high level of methylation detected at some of these mCs may simply reflect the overall low number of reads at these sites.

\section{Comparative analysis between replicate methylomes}

In an effort to examine the Dictyostelium methylome profile in more detail, we performed a replicate of the entire experiment. The overall results for the second-round of whole genome methylation analysis were comparable to the first-round results (see Table 3 for comparative summary. The same general characteristics for the two replicate methylomes were observed, including a very low level of methylation with just 462 and 459 significantly methylated cytosines, respectively, in the entire genome (Table 3). Surprisingly, 51 of the mCs were shared between the two replicates, including $5 \mathrm{mCGs}, 2 \mathrm{mCHGs}$, and $44 \mathrm{mCHHs}$ (Table 4), representing $11.04 \%$ of all the mCs. At the vast majority of these 51 individual $\mathrm{mC}$ sites, the level of methylation is above $10 \%$ and the total number of reads is greater than 10 in both replicates (Table 4). These 51 robust $\mathrm{mCs}$ are widely dispersed across the Dictyostelium genome, with numerous $\mathrm{mCs}$ found on each of the six chromosomes and in the mitochondrial DNA (Fig. 5). An additional feature found amongst the $\mathrm{mCHH}$ sites is that 20 of the 44 methylated cytosines are found in close proximity pairs (shown in gray in Table 4, Table S2).

\section{Robust $\mathrm{mC}$ genomic features}


Of the 51 robust mCs, 11 are located directly in annotated genes (Table S3). Gene Ontology (GO) analysis of these $11 \mathrm{mCs}$ revealed enrichment in genes involved in the generation of precursor metabolites and energy (GO:0006091), driven by the nad1 (DDB_GO294018), nad11 (DDB_GO294052), and atp1 (DDB_G00294012) genes. Contrary to an earlier study [20], no evidence of DNA methylation at the guaB gene could be detected in our analysis. The nearest $\mathrm{mC}$ to guaB is over 1.5 million base pairs away, suggesting that this gene is not a target for methylation in late development. Intriguingly, a single methylated site is detected in the DIRS1 retrotransposon on chromosome 1 (Table S3), supporting the previous observation of methylation in this class of retrotransposable element [20].

Examining the genomic neighborhoods around the 51 robust $\mathrm{mCs}$, there are a total of 98 genes located within $1 \mathrm{~kb}$ either upstream or downstream of individual mCs (Table S4). Amongst these genes, no single GO term is enriched and a wide range of protein classes are represented, including enzymes potentially involved in metabolic pathways (hydrolases, oxireductases and transferases) and nucleic acid binding proteins (Fig. 6). Given that our analysis was performed on the Dictyostelium cells at $18-24$ hours post-starvation, it is perhaps not surprising to also find methylation proximal to, or within, genes associated with developmental-related processes such as slug and sorocarp stalk cell differentiation and morphogenesis (proA/DDB_G0287125, st/B/DDB_G0290853 and DDB_G0277555) and pheromone response (DDB_G0284821) (Table S3 and S4). A number of retrotransposable elements are also located within $1 \mathrm{~kb}$ of a $\mathrm{mC}$, including six from the DIRS1 family and three from the TRE family (Table S4), supporting a possible role for DNA methylation in the silencing of retrotransposons, as has been previously reported [18]. In addition, six different tRNA-encoding genes (DDB_G0294885, DDB_G0295189, DDB_G0294641, $D D B_{-} G 0294643, D D B_{-} G 0294645$, and DDB_G0294657) are located close to mCs (Table S4). 


\section{Conclusions}

The paucity of studies investigating DNA methylation in $D$. discoideum has prohibited our detailed understanding of the evolution and function of eukaryotic DNA methylation with reference to $D$. discoideum's unique phylogenetic position. That is, $D$. discoideum diverged soon after the plant-animal split and is therefore closely related to two kingdoms with largely present or absent DNA methylation systems (i.e., plants and fungi, respectively) [17] (Fig. 1). In our current study utilizing deep sequencing, we demonstrate that $D$. discoideum does possess a rudimentary DNA methylation system, as evidenced by overall very low, but reproducible levels of methylation in the genome. Our findings support the earlier observation that the genome of D. discoideum is largely devoid of methylation [19], but a few sites show robust, replicable patterns of methylation. Amongst these sites are $\mathrm{mCs}$ in, or in close proximity to, annotated transposable elements, tRNA genes and genes encoding for proteins with metabolic and developmental pathways [20]. Combining our new data with the knowledge that there is a functional DNMT2-homolog (DNMA) with potentially broad substrate recognition in $D$. discoideum, opens up the exciting possibility of further dissecting this evolutionarily conserved epigenetic system in future studies. 


\section{Methods}

\section{DNA Sources}

To investigate the potential for DNA methylation sites in D. discoideum, AX4 strain cells were selected for whole genome analysis. D. discoideum AX4s have near-complete chromosome level genome assembly with genes functionally validated or predicted, making it the ideal and only current candidate for whole genome analysis. D. discoideum AX4s were grown in HL5 liquid cultures (dictybase.org for recipe) until near saturation. Once sufficient cell density was reached, $30 \mathrm{~mL}$ of the culture was transferred to $50 \mathrm{~mL}$ conical tubes. The cells were centrifuged for 5 minutes at 1000RPM, room temperature, the media was aspirated from the tube, and the cells were resuspended in $1.5 \mathrm{~mL} 1 \mathrm{x}$ Starvation Buffer (dictybase.org). The resuspended cells were transferred to nitrocellulose membrane pads with $0.45 \mu \mathrm{m}$ pores pre-wetted with Starvation Buffer, and allowed to develop over the course of 18 to 24 hours. After this time, the cells were scraped from the pads, and gDNA was extracted with a Genesee Scientific ZR Genomic DNA Tissue MiniPrep extraction kit, utilizing the Solid Tissue protocol. After extraction, bisulfite sequencing was conducted to determine potential sites of methylation in the genome.

\section{Sequencing of Bisulfite Converted DNA Libraries}

Library construction, bisulfiite conversion and sequencing were performed at the Beijing Genomics Institute. Briefly, DNA was fragmented into 100-300 bp fragments by sonication (Covaris S-2, Woburn USA). The fragmentation parameters were: Duty cycle 10\%; Intensity: 5; Cycles/burst: 200; Cycles: 16; Total fragmentation time: $960 \mathrm{sec}$. Fragmentation was confirmed using a 2100 Bioanalyzer (Aligent Technologies, Santa Clara, USA). Fragments were end repaired (Illumina) as recommended by the manufacturer. Repaired fragments were ligated with methylated sequencing adaptors using a paired end adaptor oligo kit and oligo mix 5 (Illumina). Ligated fragments were selected by gel electrophoresis and fragments of size 360 bp extracted using a QIAquick gel extraction kit (Qiagen).

Size-selected fragments were bisulfite treated using an EZ-DNA methylation kit (Zymo Research, Irvine USA) and enriched using a MethyMiner methylated DNA enrichment kit (Invitrogen). It should be noted that this kit uses the DNA binding domain from human methyl-binding domain 2 protein to enrich for methylated DNA and therefore, when compared to experimental 
approaches that do not use this step, it will likely introduce selection for methylated fragments. Libraries were amplified using T4 polymerase (Enzymatics), and sequenced on Illumina's HiSeq PE 150 platform.

\section{Sequence Analysis and Mapping DNA Methylation}

Data were filtered to remove adaptor sequences, duplicate sequences, contamination and low quality reads using BGI software. We mapped our reads onto the Dictyostelium discoideum genome assembly 1.0 [17] using BSMAP version 2.6 [26] with seed size 12 and maximum allowed mismatches 5. Similarly, we mapped our reads onto the complete lambda phage genome (GenBank: J02459.1). 72.53\% of our reads mapped onto the Dictyostelium genome. We considered only reads that mapped uniquely, and bases within reads that had a quality score of 20 or more, and that were next to 3 matches with quality scores of at least 15 [27]. From these data, we determined the number of converted and unconverted reads at each cytosine position in the Dictyostelium and lambda genome assemblies, accounting for the fact that each read comes from a bisulfite reaction on one strand or the other.

To estimate the overall rate of bisulfite conversion in non-methylated bases in our experiments, we used the $\mathrm{C}$ to $\mathrm{T}$ conversion rate in the lambda phage DNA, in which all cytosines should have been converted. We found that $99.44 \%$ of cytosines were converted in the lambda DNA, indicating that our false negative rate is less than $0.6 \%$.

The average methylation level by chromosome was determined by the ratio of the number of reads supporting methylation to the number of reads covering a particular cytosine site:

$$
R m_{\text {average }}=\frac{N_{m}}{N_{m}+N_{n m}} \times 100 \%
$$

Where $\mathrm{N}_{\mathrm{m}}$ represents the number of methyl-C (non-converted) reads and $\mathrm{N}_{\mathrm{nm}}$ represents the number of non-methylated (converted) reads.

To identify individual cytosines that were significantly methylated in the Dictyostelium genome, we compared the number of converted and non-converted reads at each site. We used only sites that had coverage of 4 or more and no more than 1000 reads. We asked how likely these 
counts were under a binomial test where the probability of success is one minus the conversion rate, and corrected this probability value for multiple testing [28], as previously described [24, $25,29]$. From this, were able to determine the methylated sites in the genome and the level of methylation at individual 5-methylcytosines.

\section{Gene Ontology Enrichment and Protein Class Mapping}

To determine what functional categories are overrepresented among methylated sites of genes either directly methylated or within $1 \mathrm{~kb}$ of a methylated site, a list of genes fitting these criteria were identified using BEDTools, version 2.25 [30]. The list of genes was then inputted to AmiGO2, version 2.4.24 [31] using the PANTHER, version 11.1 [32], overrepresentation test (release 20160715). Similarly, to summarize the protein classes of genes within $1 \mathrm{~kb}$ of a methylated site, PANTHER's functional classification function was used. 


\section{Figures}

\section{Figure 1. Evolution of DNA methyltransferases}

The presence (dark boxes) or absence (white boxes) of biologically significant DNA methylation and the DNA methyltransferase (DNMT) enzymes across eukaryotes. Kingdom, Animalia: $H$. sapiens, Homo sapiens; D. melanogaster, Drosophila melanogaster; A. mellifera, Apis mellifera. Kingdom, Fungi: S. cerevisiae, Saccharomyces cerevisiae; N. crassa, Neurospora crassa; S. pombe, Schizosaccharomyces pombe. Kingdom, Protozoa: D. discoideum, Dictyostelium discoideum. Kingdom Plantae: Z. mays, Zea mays subsp. mays; O. sativa, Oryza sativa; A. thaliana, Arabidopsis thaliana. The tree was generated using the NCBI taxonomy common tree (https://www.ncbi.nlm.nih.gov/Taxonomy/CommonTree/wwwcmt.cgi).

Figure 2. Sequencing depth and cumulative coverage of the Dictyostelium methylome.

(A) Sequencing depth distribution across the entire genome. (B) Cumulative coverage across the entire genome, e.g. approximately $90 \%$ of sites are covered by 100 or more reads. (C) Percentage of cytosines that have a certain level of coverage, e.g. approximately $80 \%$ of sites have coverage of 75 reads. The profile for cytosines in different sequence contexts (CG, CHG and $\mathrm{CHH}$, where $\mathrm{H}=$ non- $\mathrm{G}$ base) was similar.

\section{Figure 3. Overall methylation level at methyl-cytosines}

The methylation level at the 462 individual mCs is calculated and organized in $10 \%$ bins (i.e. 0$10 \%, 91-100 \%$ etc.). Only mCs covered by at least four and no more than 1000 reads are used in this calculation. The vast majority of $\mathrm{mCs}$, irrespective of sequence context, have less than $10 \%$ methylation level.

\section{Figure 4. Methylation level and read depth at individual $\mathrm{mCs}$}

(A) The methylation level at the 462 individual $\mathrm{mCs}$ is depicted for each of the six genomic chromosomes and the mitochondrial (MT) DNA. Only mCs covered by at least four and no more than 1000 reads are shown. (B) The total read number for the 462 individual methylated sites. The vast majority of $\mathrm{mCs}$, irrespective of sequence context, have less than 100 total reads. (C) and (D) The methylation level (\%) plotted against the total read number for the 462 individual methylated sites demonstrates a clear inverse relationship between read depth and methylation 
level at the mCs, with sites showing higher levels of methylation having lower read depth and vice-versa. All the methylated sites in the genome with a methylation higher than $40 \%$ have fewer than 25 total reads.

\section{Figure 5. Genomic location of robust methylated sites}

The six nuclear chromosomes and mitochondrial genome (10x enlarged) are depicted with plus strand (left side) and minus (right side) genes indicated (grey boxes). Diamonds depict the location of the robust CG (red), CHG (blue), and CHH (green) methylated sites detected in the replicate experiments. These methylated sites are distributed throughout the genome and occur predominantly at $\mathrm{CHH}$ sequences.

\section{Figure 6. Protein classes of genes within $1 \mathrm{~kb}$ of a robust methylated site}

The frequency of different GO protein classes for the annotated genes located within $1 \mathrm{~kb}$ of a robust methylated site are depicted. Methylated sites were most often associated with genes encoding for hydrolases (PC00121) and nucleic acid binding (PC00171). 


\section{Tables}

\section{Table 1. Methylation level by chromosome}

The values represent the average methylation level at cytosine bases on each chromosome and the mitochondrial DNA (chrMT). The percentage value was determined by the ratio of the number of reads supporting methylation to the number of reads covering a particular cytosine site (see methods for details). The profile for cytosines in different sequence contexts (CG, CHG and $\mathrm{CHH}$, where $\mathrm{H}=$ non- $\mathrm{G}$ base) is shown. It should be noted that the calculated bisulfite conversion rate in the experiment was $99.44 \%$.

\section{Table 2. Methylated cytosines in CG, CHG and $\mathrm{CHH}$ genomic sequence context}

Methylation occurs predominantly at $\mathrm{CHH}$ sites, but is also detectable at $\mathrm{CG}$ and $\mathrm{CHG}$ sites. Of the approximately 7.5 million cytosines in the genome, only $462(0.006 \%$ of all sites) are significantly methylated.

\section{Table 3. Comparison between the two replicate methylomes}

Key metrics for the replicate methylomes. The overall profile is similar in both experiments.

\section{Table 4. Shared mCGs, mCHGs and mCHHs}

The methylated sites identified in both replicate experiments are listed, including 5 mCGs, 2 mCHGs and $44 \mathrm{mCHHs}$, by genomic location, coordinate, total read number and methylation level. Many of these robust mCs show methylation levels above $10 \%$ in both experiments. Amongst the $\mathrm{mCHHs}, 20$ are found in close proximity pairs (shaded boxes). 


\section{Supporting Information}

\section{Supplementary Table 1. Cytosine coverage by chromosome}

The values represent the average coverage at cytosine bases on each chromosome and the mitochondrial DNA (chrMT). The profile for cytosines in different sequence contexts (CG, CHG and $\mathrm{CHH}$, where $\mathrm{H}=$ non- $\mathrm{G}$ base) is shown.

\section{Supplementary Table 2. Robust $\mathrm{mCHH}$ proximity pairs GO classification}

The chromosomal location and identity of different GO protein classes for the annotated genes located within $1 \mathrm{~kb}$ of a robust methylated $\mathrm{CHH}$ proximity pair are listed.

\section{Supplementary Table 3. 11 robust $\mathrm{mCs}$ are located directly in genes}

The chromosomal location, sequence context and identity for the annotated genes are shown.

\section{Supplementary Table 4 . Genes within $1 \mathrm{~kb}$ window of a robust $\mathrm{mC}$}

The chromosomal location, sequence context and identity for the annotated genes within $1 \mathrm{~kb}$ of a robust methylated cytosine are listed. 


\section{Availability of Supporting Data}

The data set supporting the results of this article is available at:

Will be publicly released at publication

\section{Abbreviations}

DNMT = DNA methyltransferase

$\mathrm{GO}=$ Gene ontology

\section{Competing interests}

The authors report no competing interests.

\section{Authors' contributions}

JLS conceived of the study, participated in the design of the study and helped to draft the manuscript. JSD performed the gDNA preparation and drafted the manuscript. JMD carried out sequence and bioinformatic analysis. DAL conceived of the study, participated in the design of the study and helped to draft the manuscript. RAD conceived of the study, participated in its design, established the genomics pipeline, coordinated and drafted the manuscript. All authors read and approved the final manuscript.

\section{Acknowledgements}

This work was funded in part by a National Institutes of Health (GM110571) grant to RAD and JMD. 


\section{References}

1. Suzuki MM, Bird A: DNA methylation landscapes: provocative insights from epigenomics. Nat Rev Genet 2008, 9:465-476.

2. Schubeler D: Function and information content of DNA methylation. Nature 2015, 517:321-326.

3. Challen GA, Sun D, Jeong M, Luo M, Jelinek J, Berg JS, Bock C, Vasanthakumar A, Gu H, Xi $Y$, et al: Dnmt3a is essential for hematopoietic stem cell differentiation. Nat Genet 2011, 44:23-31.

4. Li E, Beard C, Jaenisch R: Role for DNA methylation in genomic imprinting. Nature 1993, 366:362-365.

5. Putiri EL, Robertson KD: Epigenetic mechanisms and genome stability. Clin Epigenetics 2011, 2:299-314.

6. Hellman A, Chess A: Gene body-specific methylation on the active $\mathbf{X}$ chromosome. Science 2007, 315:1141-1143.

7. Bestor TH: The DNA methyltransferases of mammals. Hum Mol Genet 2000, 9:23952402.

8. Goll MG, Bestor TH: Eukaryotic cytosine methyltransferases. Annu Rev Biochem 2005, 74:481-514.

9. Chedin F: The DNMT3 family of mammalian de novo DNA methyltransferases. Prog Mol Biol Transl Sci 2011, 101:255-285.

10. Dong A, Yoder JA, Zhang X, Zhou L, Bestor TH, Cheng X: Structure of human DNMT2, an enigmatic DNA methyltransferase homolog that displays denaturant-resistant binding to DNA. Nucleic Acids Res 2001, 29:439-448.

11. Schaefer M, Lyko F: Solving the Dnmt2 enigma. Chromosoma 2010, 119:35-40.

12. Schaefer M, Pollex T, Hanna K, Tuorto F, Meusburger M, Helm M, Lyko F: RNA methylation by Dnmt2 protects transfer RNAs against stress-induced cleavage. Genes Dev 2010, 24:1590-1595.

13. Phalke S, Nickel O, Walluscheck D, Hortig F, Onorati MC, Reuter G: Retrotransposon silencing and telomere integrity in somatic cells of Drosophila depends on the cytosine-5 methyltransferase DNMT2. Nat Genet 2009, 41:696-702. 
14. Jeltsch A, Nellen W, Lyko F: Two substrates are better than one: dual specificities for Dnmt2 methyltransferases. Trends Biochem Sci 2006, 31:306-308.

15. Lyko F, Ramsahoye BH, Jaenisch R: DNA methylation in Drosophila melanogaster. Nature 2000, 408:538-540.

16. Raddatz G, Guzzardo PM, Olova N, Fantappie MR, Rampp M, Schaefer M, Reik W, Hannon GJ, Lyko F: Dnmt2-dependent methylomes lack defined DNA methylation patterns. Proc Natl Acad Sci U S A 2013, 110:8627-8631.

17. Eichinger L, Pachebat JA, Glockner G, Rajandream MA, Sucgang R, Berriman M, Song J, Olsen $R$, Szafranski $K, X u Q$, et al: The genome of the social amoeba Dictyostelium discoideum. Nature 2005, 435:43-57.

18. Kuhlmann M, Borisova BE, Kaller M, Larsson P, Stach D, Na J, Eichinger L, Lyko F, Ambros $\mathrm{V}$, Soderbom $\mathrm{F}$, et al: Silencing of retrotransposons in Dictyostelium by DNA methylation and RNAi. Nucleic Acids Res 2005, 33:6405-6417.

19. Smith SS, Ratner DI: Lack of 5-methylcytosine in Dictyostelium discoideum DNA. Biochem J 1991, 277 ( Pt 1):273-275.

20. Katoh M, Curk T, Xu Q, Zupan B, Kuspa A, Shaulsky G: Developmentally regulated DNA methylation in Dictyostelium discoideum. Eukaryot Cell 2006, 5:18-25.

21. Muller S, Windhof IM, Maximov V, Jurkowski T, Jeltsch A, Forstner KU, Sharma CM, Graf R, Nellen W: Target recognition, RNA methylation activity and transcriptional regulation of the Dictyostelium discoideum Dnmt2-homologue (DnmA). Nucleic Acids Res 2013, 41:8615-8627.

22. Li Y, Tollefsbol TO: DNA methylation detection: bisulfite genomic sequencing analysis. Methods Mol Biol 2011, 791:11-21.

23. Lister R, Pelizzola M, Dowen RH, Hawkins RD, Hon G, Tonti-Filippini J, Nery JR, Lee L, Ye Z, Ngo QM, et al: Human DNA methylomes at base resolution show widespread epigenomic differences. Nature 2009, 462:315-322.

24. Beeler SM, Wong GT, Zheng JM, Bush EC, Remnant EJ, Oldroyd BP, Drewell RA: Wholegenome DNA methylation profile of the jewel wasp (Nasonia vitripennis). G3 (Bethesda) 2014, 4:383-388.

25. Drewell RA, Bush EC, Remnant EJ, Wong GT, Beeler SM, Stringham JL, Lim J, Oldroyd BP: The dynamic DNA methylation cycle from egg to sperm in the honey bee Apis mellifera. Development 2014, 141:2702-2711. 
26. $\mathrm{Xi}$ Y, Li W: BSMAP: whole genome bisulfite sequence MAPping program. $B M C$ Bioinformatics 2009, 27:10:232.

27. Altshuler D, Pollara VJ, Cowles CR, Van Etten WJ, Baldwin J, Linton L, Lander ES: An SNP map of the human genome generated by reduced representation shotgun sequencing. Nature 2000, 407:513-516.

28. Benjamini $\mathrm{Y}$, Hochberg $\mathrm{Y}$ : Controlling the false discovery rate: A practical and powerful approach to multiple testing. Journal of the Royal Statistical Society B 1995, 57:289-300.

29. Remnant EJ, Ashe A, Young PE, Buchmann G, Beekman M, Allsopp MH, Suter CM, Drewell RA, Oldroyd BP: Parent-of-origin effects on genome-wide DNA methylation in the Cape honey bee (Apis mellifera capensis) may be confounded by allele-specific methylation. BMC Genomics 2016, 17:226.

30. Quinlan AR, Hall IM: BEDTools: a flexible suite of utilities for comparing genomic features. Bioinformatics 2010, 26:841-842.

31. Carbon S, Ireland A, Mungall CJ, Shu S, Marshall B, Lewis S: AmiGO: online access to ontology and annotation data. Bioinformatics 2009, 25:288-289.

32. Thomas PD, Campbell MJ, Kejariwal A, Mi H, Karlak B, Daverman R, Diemer K, Muruganujan A, Narechania A: PANTHER: a library of protein families and subfamilies indexed by function. Genome Res 2003, 13:2129-2141. 


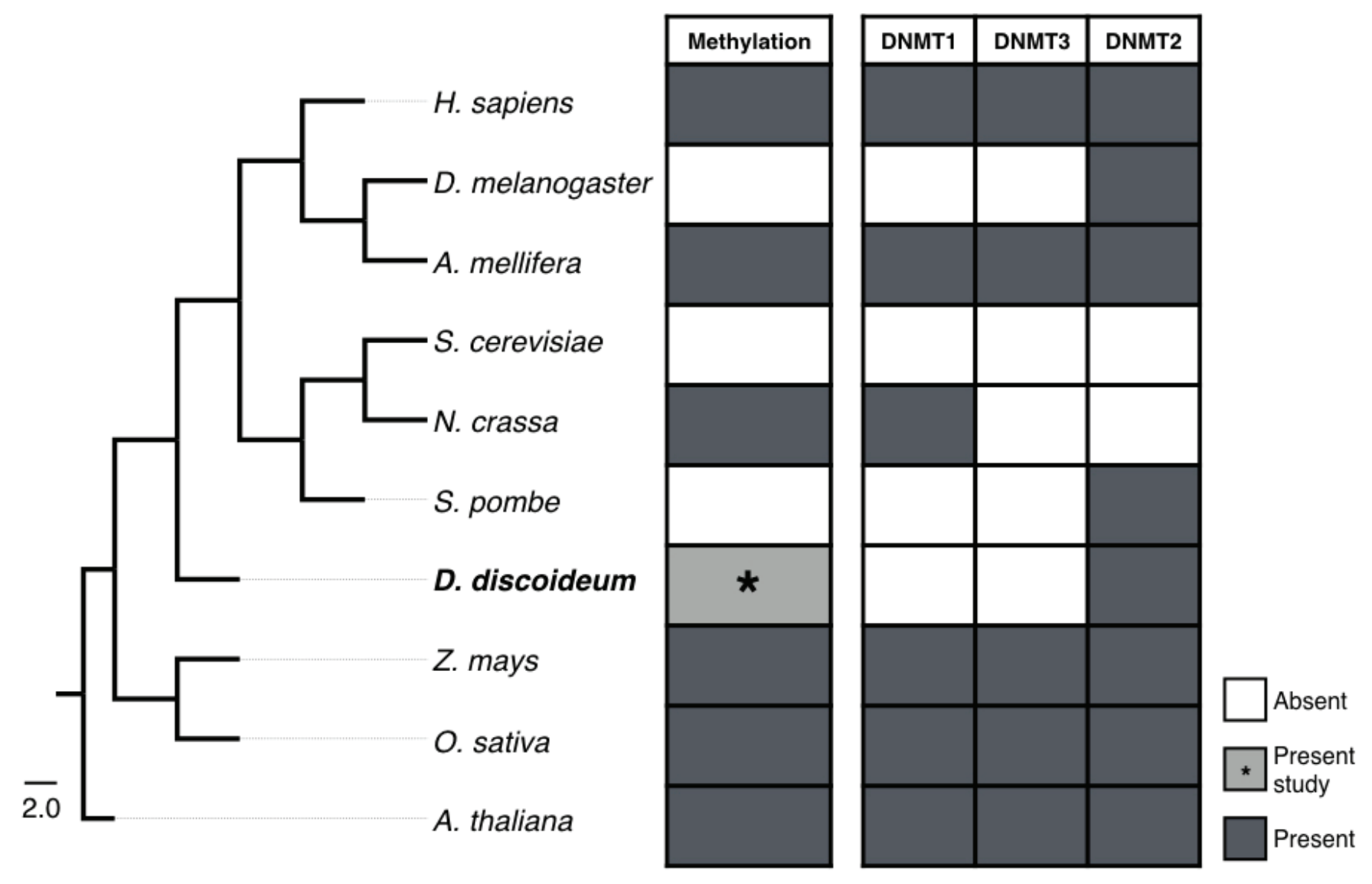


A

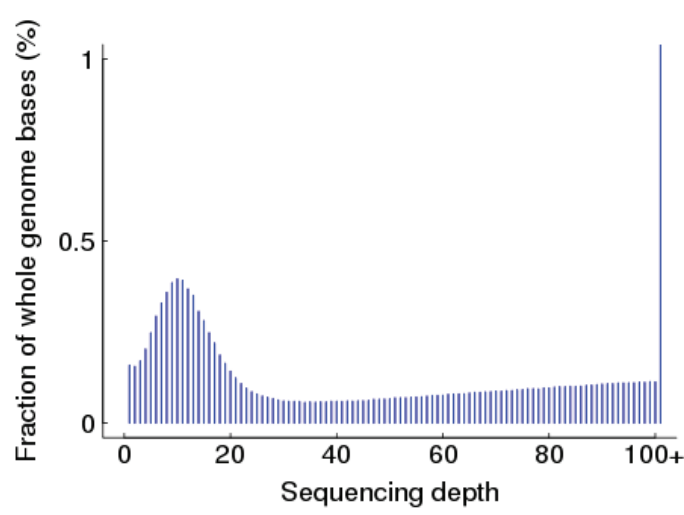

B

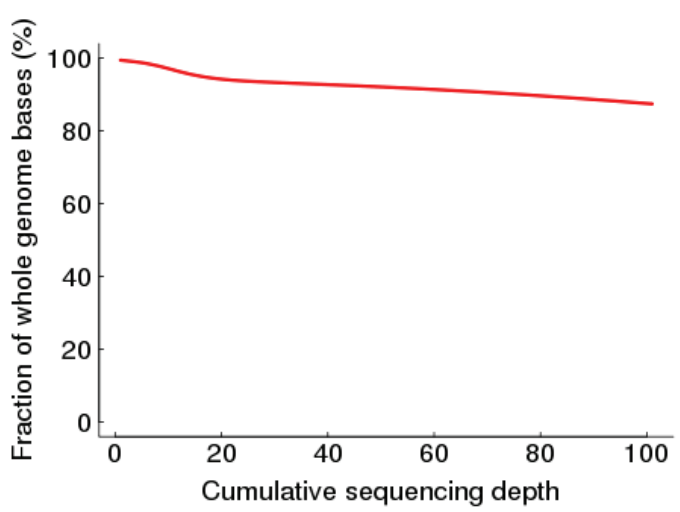

C

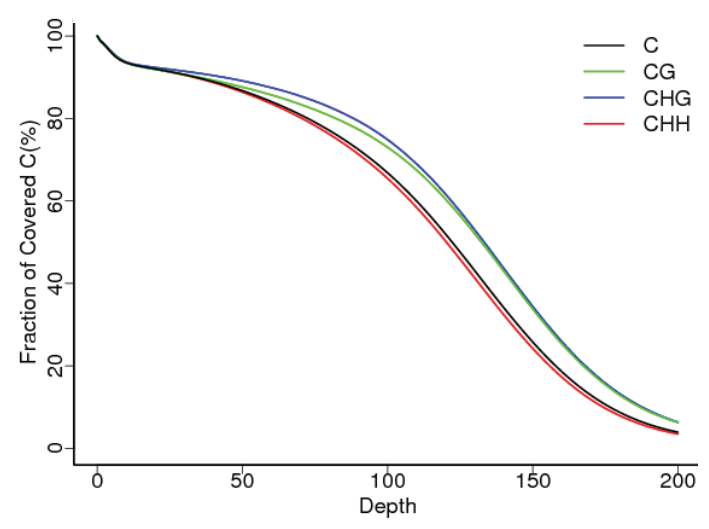




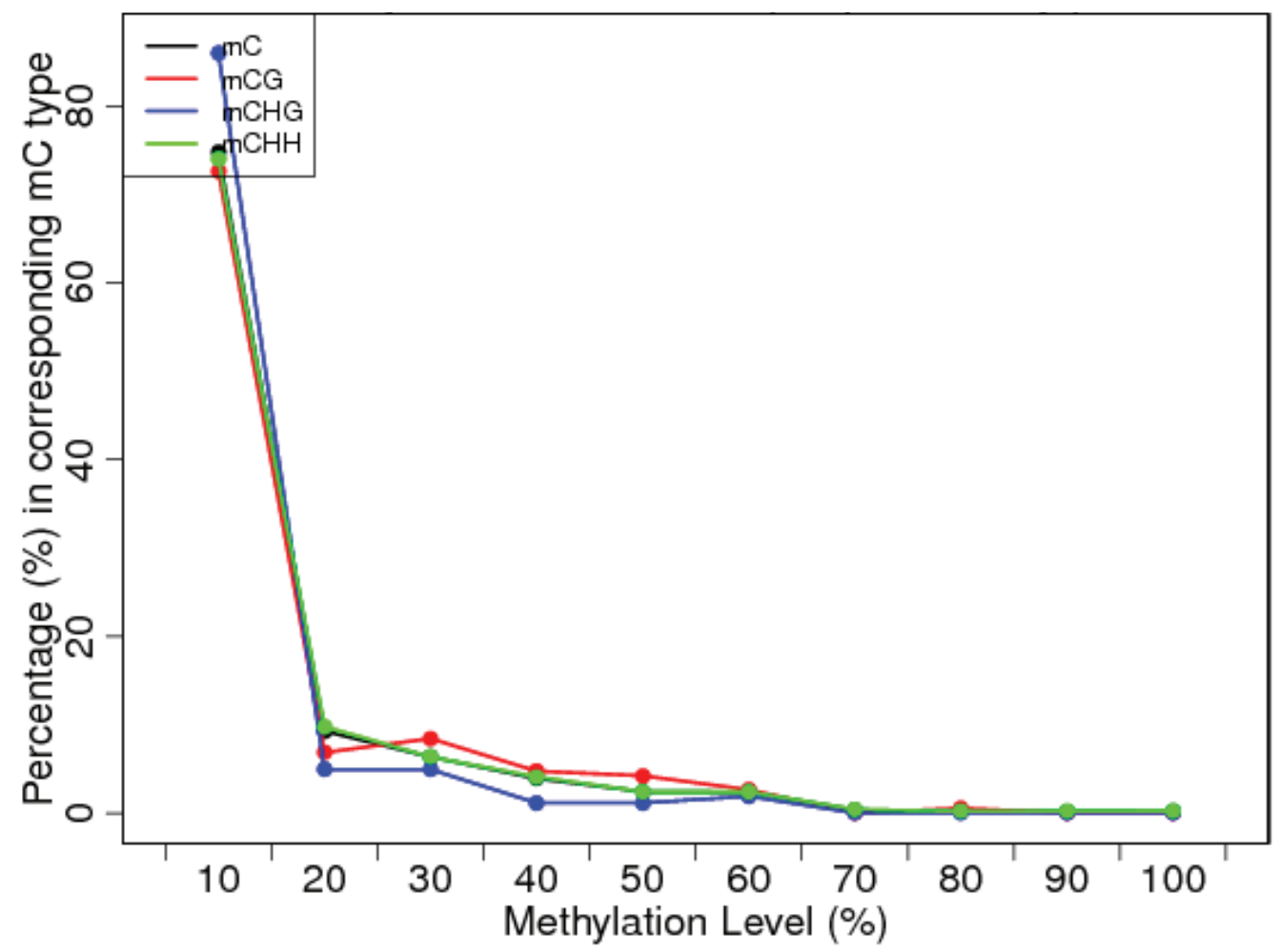


A

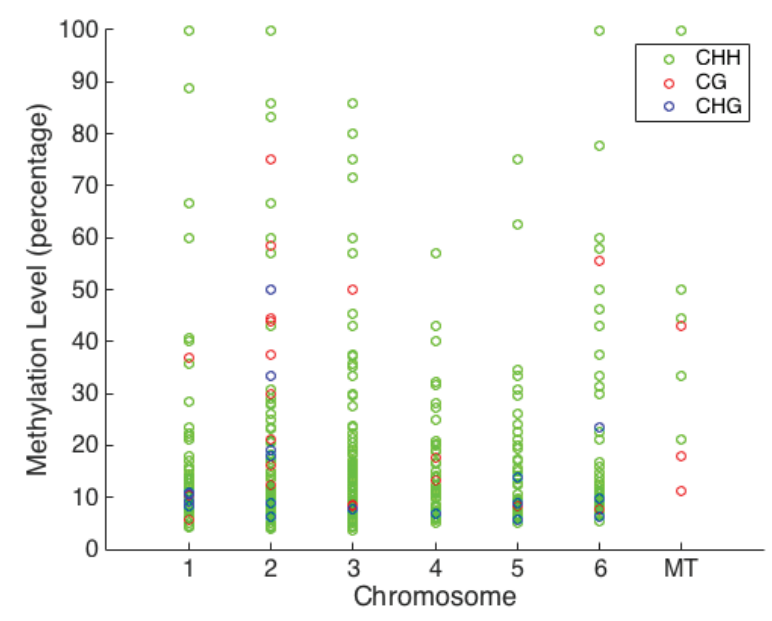

C

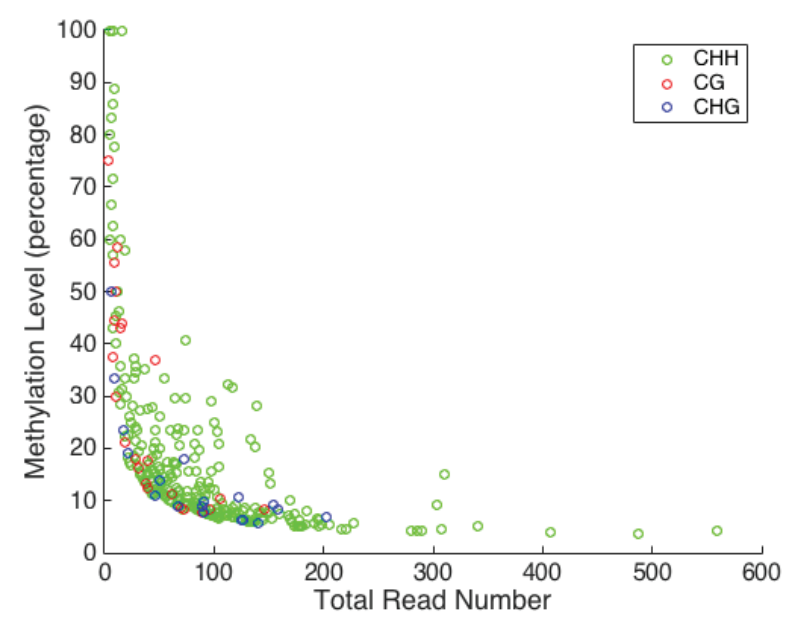

B

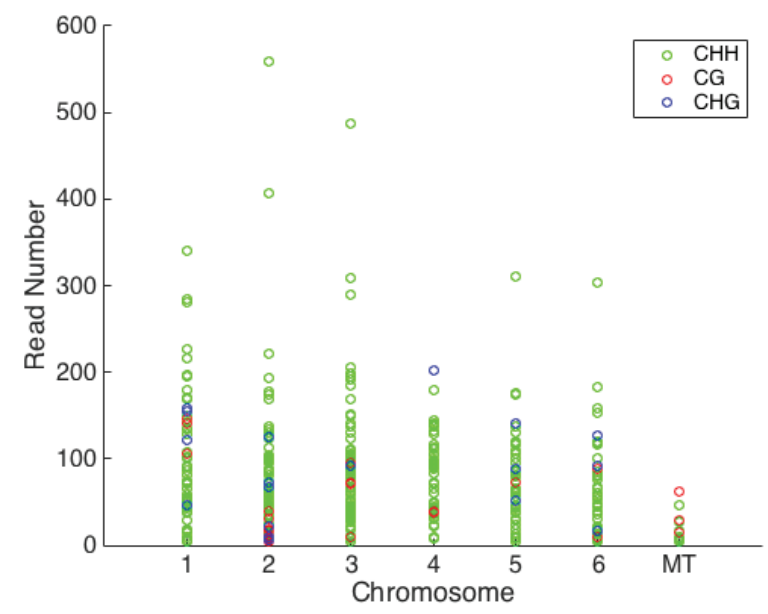

D

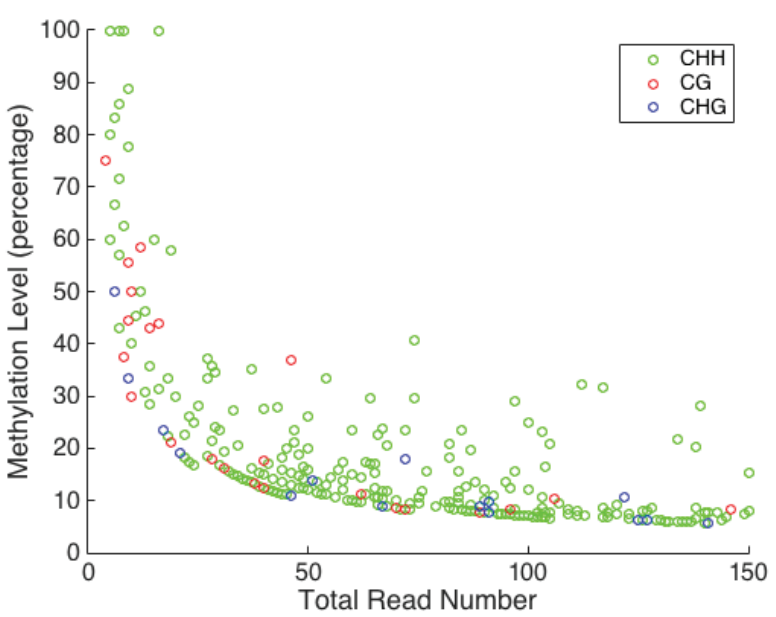




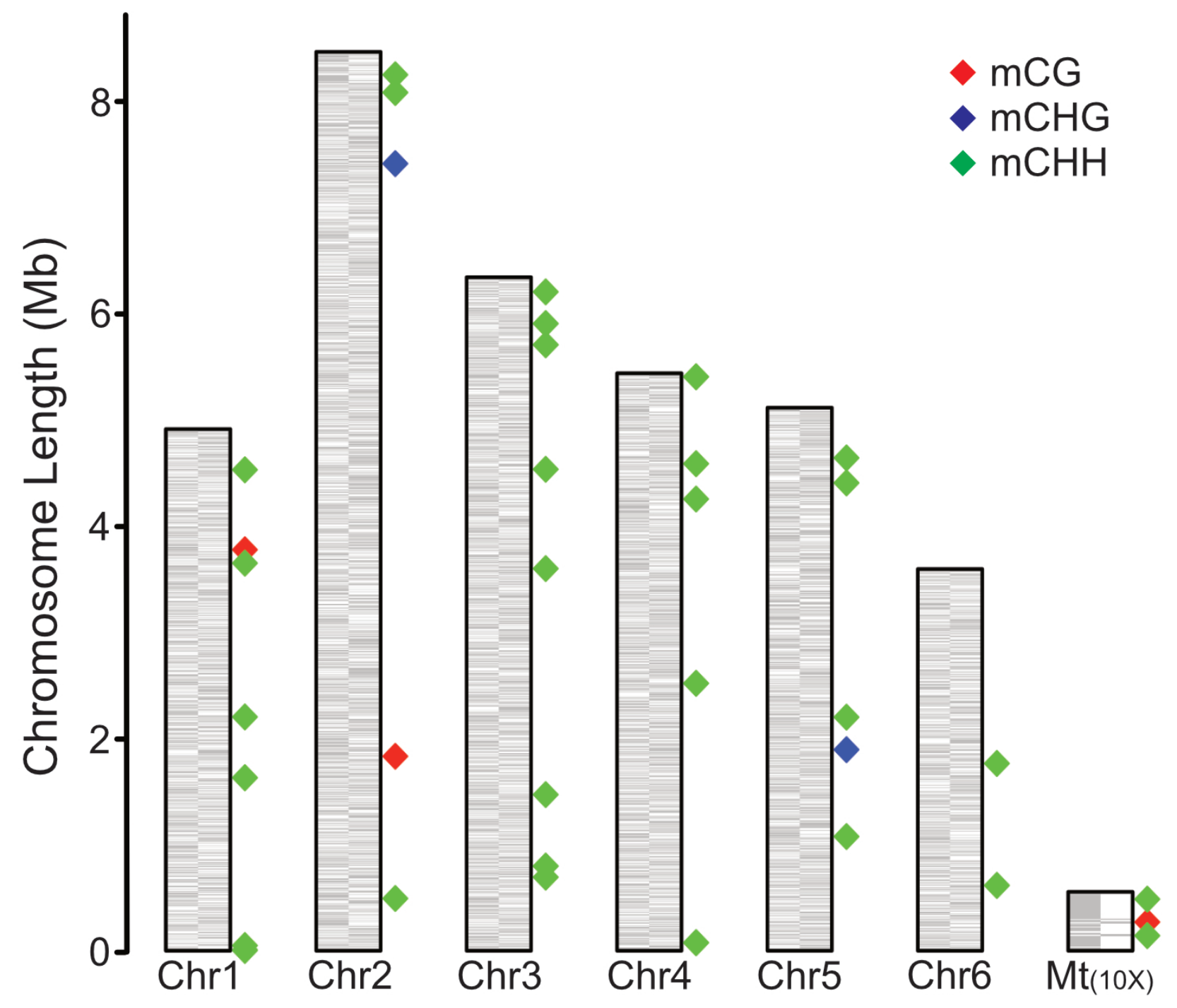




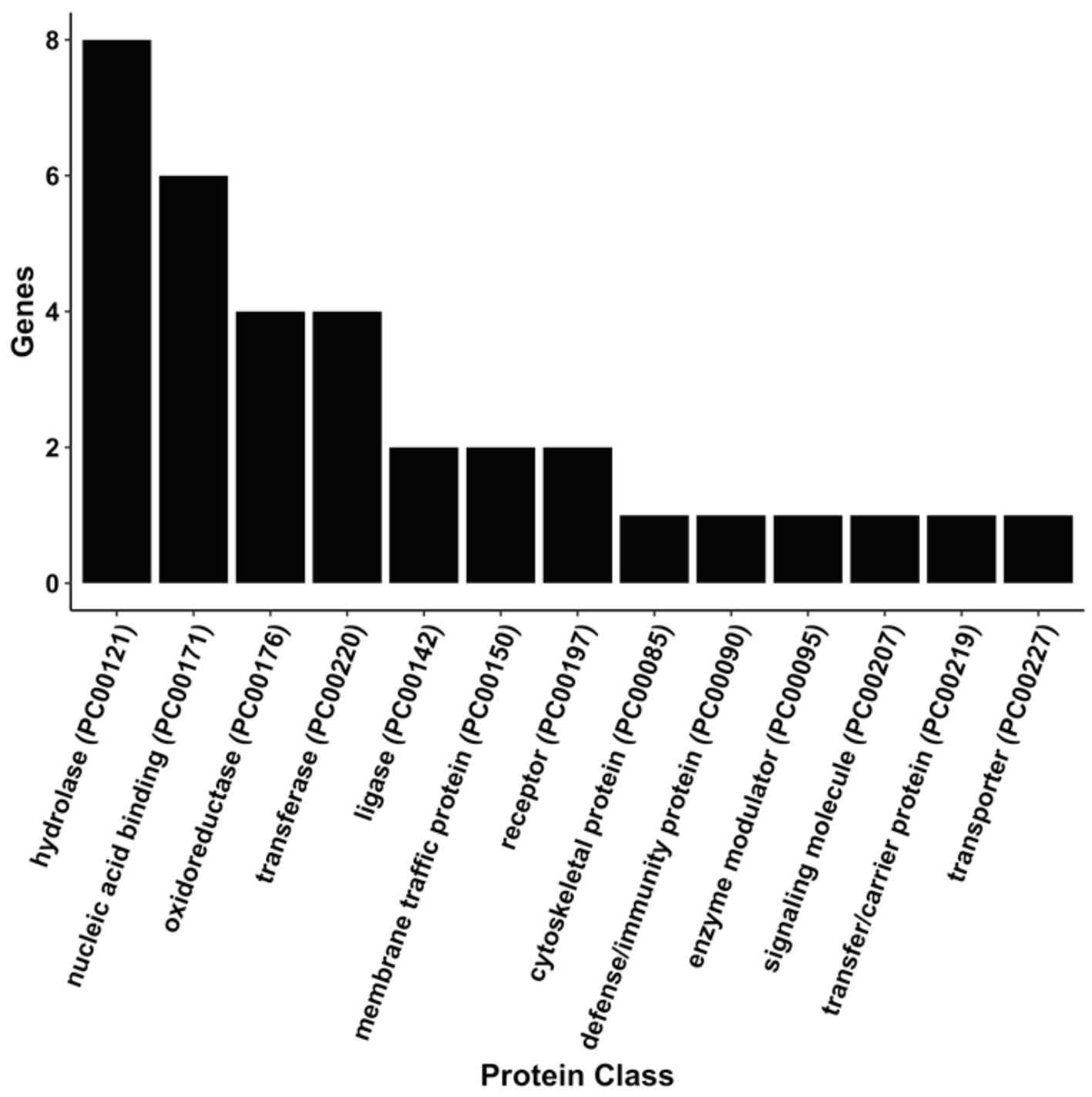




\begin{tabular}{|lcccc|}
\hline Chromosome & C & CG & CHG & CHH \\
\hline chr1 & 0.519 & 0.438 & 0.481 & 0.531 \\
chr2 & 0.525 & 0.435 & 0.484 & 0.537 \\
chr3 & 0.524 & 0.439 & 0.484 & 0.536 \\
chr4 & 0.523 & 0.434 & 0.487 & 0.535 \\
chr5 & 0.521 & 0.432 & 0.484 & 0.532 \\
chr6 & 0.521 & 0.435 & 0.481 & 0.533 \\
chrMT & 0.528 & 0.46 & 0.499 & 0.546 \\
Whole Genome & 0.523 & 0.442 & 0.483 & 0.537 \\
\hline
\end{tabular}




\begin{tabular}{|lcccc|}
\hline & mCG & mCHG & mCHH & mC \\
\hline mC reads & 180 & 161 & 3,431 & 3,772 \\
proportion (\%) & 4.772 & 4.268 & 90.960 & 100 \\
& & & & \\
mC sites & 27 & 19 & 416 & 462 \\
proportion (\%) & 5.844 & 4.113 & 90.043 & 100 \\
& & & & \\
total C number & 442,821 & 733,151 & $6,317,717$ & $7,493,689$ \\
proportion $\mathrm{mC}$ (\%) & 0.006 & 0.003 & 0.007 & 0.006 \\
\hline
\end{tabular}




\begin{tabular}{|lcc|}
\hline & First-round & Second-round \\
\hline Clean reads & $125,357,640$ & $73,253,322$ \\
Mapping rate (\%) & 72.53 & 67.96 \\
Bisulfite conversion rate (\%) & 99.44 & 99.50 \\
Genome coverage (x) & 286.14 & 149.58 \\
Cytosine coverage (\%) & 98.816 & 97.688 \\
Overall methylation level (\%) & 0.523 & 0.564 \\
mC sites & 462 & 459 \\
mCG sites & 27 & 19 \\
mCHG sites & 19 & 24 \\
mCHH sites & 416 & 416 \\
\hline
\end{tabular}




\begin{tabular}{|c|c|c|c|c|c|}
\hline $\begin{array}{l}\text { Chromosome / } \\
\text { Contig }\end{array}$ & $\begin{array}{c}\text { Genomic } \\
\text { Coordinate }\end{array}$ & $\begin{array}{l}\text { First-round } \\
\text { Total Reads }\end{array}$ & Methyl Level (\%) & $\begin{array}{c}\text { Second-round } \\
\text { Total Reads }\end{array}$ & Methyl Level (\%) \\
\hline \multicolumn{6}{|l|}{ CG } \\
\hline 1 & 3783605 & 46 & 36.96 & 9 & 44.44 \\
\hline 2 & 1836074 & 12 & 58.33 & 4 & 75.00 \\
\hline 4 & 4598084 & 38 & 13.16 & 10 & 30.00 \\
\hline MT & 28028 & 14 & 42.86 & 10 & 30.00 \\
\hline Unmapped 13 & 3627 & 73 & 15.07 & 5 & 100.00 \\
\hline \multicolumn{6}{|l|}{ CHG } \\
\hline 2 & 7430589 & 72 & 18.06 & 17 & 35.29 \\
\hline 5 & 1898452 & 89 & 8.99 & 20 & 25.00 \\
\hline \multicolumn{6}{|l|}{$\mathrm{CHH}$} \\
\hline 1 & 3379 & 195 & 5.13 & 55 & 10.91 \\
\hline 1 & 9597 & 196 & 6.12 & 25 & 20.00 \\
\hline 1 & 49448 & 171 & 5.85 & 22 & 18.18 \\
\hline 1 & 1633944 & 134 & 21.64 & 53 & 15.09 \\
\hline 1 & 1633981 & 150 & 15.33 & 65 & 23.08 \\
\hline 1 & 2207539 & 74 & 40.54 & 17 & 23.53 \\
\hline 1 & 3659412 & 65 & 16.92 & 24 & 37.50 \\
\hline 1 & 4540349 & 28 & 17.86 & 17 & 29.41 \\
\hline 2 & 493889 & 68 & 20.59 & 12 & 33.33 \\
\hline 2 & 8101959 & 85 & 23.53 & 23 & 26.09 \\
\hline 2 & 8102002 & 97 & 28.87 & 20 & 20.00 \\
\hline 2 & 8269415 & 44 & 18.18 & 15 & 33.33 \\
\hline 3 & 695099 & 62 & 14.52 & 17 & 23.53 \\
\hline 3 & 799417 & 34 & 20.59 & 6 & 100.00 \\
\hline 3 & 799419 & 54 & 33.33 & 22 & 31.82 \\
\hline 3 & 1474373 & 90 & 12.22 & 22 & 27.27 \\
\hline 3 & 3607695 & 109 & 8.26 & 45 & 24.44 \\
\hline 3 & 4545189 & 53 & 11.32 & 17 & 23.53 \\
\hline 3 & 5720793 & 74 & 29.73 & 16 & 37.50 \\
\hline 3 & 5720812 & 84 & 11.90 & 15 & 26.67 \\
\hline 3 & 5920588 & 97 & 15.46 & 23 & 17.39 \\
\hline 3 & 5920623 & 82 & 18.29 & 20 & 20.00 \\
\hline 3 & 6220024 & 21 & 19.05 & 10 & 30.00 \\
\hline 4 & 76910 & 104 & 16.35 & 39 & 12.82 \\
\hline 4 & 76914 & 105 & 20.95 & 41 & 12.20 \\
\hline 4 & 76958 & 139 & 28.06 & 31 & 25.81 \\
\hline 4 & 76962 & 138 & 20.29 & 34 & 14.71 \\
\hline 4 & 2524803 & 112 & 32.14 & 46 & 28.26 \\
\hline 4 & 2524824 & 117 & 31.62 & 45 & 24.44 \\
\hline 4 & 4264271 & 33 & 27.27 & 5 & 60.00 \\
\hline 4 & 4598089 & 36 & 13.89 & 12 & 33.33 \\
\hline 4 & 5418388 & 90 & 10.00 & 23 & 21.74 \\
\hline 4 & 5418407 & 65 & 10.77 & 6 & 50.00 \\
\hline 5 & 1078684 & 86 & 12.79 & 16 & 31.25 \\
\hline 5 & 2205539 & 23 & 26.09 & 7 & 57.14 \\
\hline 5 & 4416786 & 311 & 15.11 & 425 & 11.29 \\
\hline 5 & 4652725 & 47 & 21.28 & 10 & 30.00 \\
\hline 6 & 617155 & 65 & 12.31 & 22 & 18.18 \\
\hline 6 & 617176 & 57 & 15.79 & 24 & 45.83 \\
\hline 6 & 1768503 & 303 & 9.24 & 465 & 4.52 \\
\hline MT & 15196 & 16 & 100.00 & 5 & 100.00 \\
\hline MT & 49661 & 9 & 44.44 & 23 & 21.74 \\
\hline Unmapped 5 & 1301 & 113 & 14.16 & 22 & 27.27 \\
\hline Unmapped 24 & 4083 & 73 & 13.70 & 8 & 75.00 \\
\hline
\end{tabular}

